\section{Association of lipoprotein subfractions and glycoprotein acetylation with coronary plaque burden in SLE}

Monica M Purmalek, ${ }^{1}$ Philip M Carlucci, ${ }^{1}$ Amit K Dey, ${ }^{2}$ Maureen Sampson, ${ }^{3}$ Yenealem Temesgen-Oyelakin, ${ }^{1}$ Simantini Sakhardande, ${ }^{1}$ Joseph B Lerman, ${ }^{2}$ Alice Fike, ${ }^{1}$ Michael Davis, ${ }^{1}$ Jonathan H Chung, ${ }^{2}$ Taufiq Salahuddin, ${ }^{2}$ Zerai Manna, ${ }^{1}$ Sarthak Gupta, ${ }^{1}$ Marcus Y Chen, ${ }^{2}$ Sarfaraz Hasni, ${ }^{1}$ Nehal N Mehta, ${ }^{2}$ Alan Remaley, ${ }^{2}$ Mariana J Kaplan ${ }^{\oplus 1}$

To cite: Purmalek MM, Carlucci PM, Dey AK, et al. Association of lipoprotein subfractions and glycoprotein acetylation with coronary plaque burden in SLE. Lupus Science \& Medicine 2019;6:e00332. doi:10.1136/ lupus-2019-000332

MMP and PMC are joint first authors.

Received 15 March 2019 Revised 9 May 2019 Accepted 18 June 2019
Check for updates

(C) Author(s) (or their employer(s)) 2019. Re-use permitted under CC BY-NC. No commercial re-use. See rights and permissions. Published by BMJ.

${ }^{1}$ National Institute of Arthritis and Musculoskeletal and Skin Diseases (NIAMS), National Institutes of Health (NIH), Bethesda, Maryland, USA ${ }^{2}$ National Heart, Lung and Blood Institute (NHLBI), National Institutes of Health, Bethesda, Maryland, USA

${ }^{3}$ National Institutes of Health Clinical Center, Bethesda, Maryland, USA

Correspondence to

Dr Mariana J Kaplan; mariana. kaplan@nih.gov

\section{ABSTRACT}

Objective Subjects with SLE display an enhanced risk of atherosclerotic cardiovascular disease (CVD) that is not explained by Framingham risk. This study sought to investigate the utility of nuclear MR (NMR) spectroscopy measurements of serum lipoprotein particle counts and size and glycoprotein acetylation (GlycA) burden to predict coronary atherosclerosis in SLE.

Methods Coronary plaque burden was assessed in SLE subjects and healthy controls using coronary CT angiography. Lipoproteins and GlycA were quantified by NMR spectroscopy.

Results SLE subjects displayed statistically significant decreases in high-density lipoprotein (HDL) particle counts and increased very low-density lipoprotein (VLDL) particle counts compared with controls. Non-calcified coronary plaque burden (NCB) negatively associated with HDL subsets whereas it positively associated with VLDL particle counts in multivariate adjusted models. GlycA was significantly increased in SLE sera compared with controls. In contrast to high-sensitivity $C$ reactive protein, elevations in GlycA in SLE significantly associated with NCB and insulin resistance (IR), though the association with NCB was no longer significant after adjusting for prednisone use.

Conclusions Patients with SLE display a proatherogenic lipoprotein profile that may significantly contribute to the development of premature CVD. The results demonstrate that NMR measures of GlycA and lipoprotein profiles, beyond what is captured in routine clinical labs, could be a useful tool in assessing CVD risk in patients with SLE.

SLE is an autoimmune disorder primarily affecting women of childbearing age that is characterised by immune dysregulation, multiorgan involvement and systemic inflammation. The Framingham Offspring Study estimates that women aged 35-44 years with SLE have a 50-fold increase in the risk of myocardial infarction compared with age-matched and gender-matched women without SLE. ${ }^{1}$ Additionally, the Framingham risk equation does not fully account for the

\section{Key messages}

Measures of glycoprotein acetylation (GlycA) and lipoprotein profiles, beyond what is captured in routine clinical labs, could be a useful tool in assessing cardiovascular risk in patients with lupus.

- In patients with lupus, GlycA is a better predictor of insulin resistance than high-sensitivity $\mathrm{C}$ reactive protein.

enhanced risk of cardiovascular disease (CVD) and CVD mortality in SLE. ${ }^{2}$

Previous studies in SLE cohorts have demonstrated that routine cholesterol measurements, including high-density lipoprotein (HDL) and low-density lipoprotein (LDL), do not differ between SLE and controls or between SLE subjects with or without coronary artery involvement. As such, CVD risk may be better explained by aberrant proatherogenic lipoprotein particle numbers. ${ }^{3-5}$ Proton nuclear MR (NMR) spectroscopy is an automated method that quantifies plasma lipoproteins and can be used to determine the size and concentration of lipoprotein particle subfractions in plasma to assess CVD. ${ }^{67}$ In addition to lipoprotein parameters, NMR also provides quantifications of serum glycoprotein acetylation (GlycA), which is an emerging composite inflammatory biomarker that predicts $\mathrm{CV}$ events in other patient populations. ${ }^{8}$

Previous studies have analysed lipoprotein particle subfractions in SLE and found no associations between these subfractions and coronary artery calcification measured via Agatston Scores. ${ }^{9}$ However, whether associations exist with non-calcified plaque burden (NCB), typically associated with higher risk of plaque rupture, remains to be determined. ${ }^{10-12}$ We now report an analysis of 
NMR determinations of lipid subfractions and GlycA and their association with subclinical coronary artery disease in SLE by assessing both calcified burden and NCB.

\section{PATIENTS AND METHODS \\ Patients}

The study protocol was conducted in accordance with principles stated in the Declaration of Helsinki. Subjects were recruited from the National Institute of Arthritis and Muskuloskeletal and Skin Diseases Lupus and Community Health Center clinics. All of the patients fulfilled revised criteria for SLE. ${ }^{13}$ Healthy adults were recruited from the National Institutes of Health healthy volunteer cohort. Detailed clinical characteristics and inclusion and exclusion criteria for this cohort have been recently described. ${ }^{14}$ In brief, 64 SLE subjects were enrolled in the study, 36 of which underwent coronary CT angiography (CCTA) as those with an estimated glomerular filtration rate $($ GFR $)<60 \mathrm{~mL} / \mathrm{min} / 1.73 \mathrm{~m}^{2}$ body surface were excluded from this assessment. A total of 30 controls were included in the study, 18 of which underwent CCTA. Homoeostatic model assessment (HOMA) was used to quantify insulin resistance (IR) $($ HOMA-IR $=(($ glucose $(\mathrm{nmol} / \mathrm{ml})+$ insulin $(\mu \mathrm{IU} /$ ml) )/22.5)).

\section{Coronary CT angiography nuclear MR}

CCTA assessment was performed as previously described. $^{14}$ Coronary plaque quantifications were controlled for the artery length by dividing total vessel plaque volume by total vessel length.

\section{Nuclear MR}

NMR assessment of lipoproteins and GlycA was performed on an FDA approved Vantera clinical NMR analyser (LabCorp, North Carolina, USA). Using the LipoProfile-3 algorithm, the average particle size and concentrations of HDL, LDL and very low density lipoprotein (VLDL) were measured (LabCorp, North Carolina, USA).

\section{Statistical analysis}

Statistical analysis was performed using STATA V.12.0 software (STATACorp, College Station, Texas, USA). A $p$ value $\leq 0.05$ was considered statistically significant. Normality was assessed on continuous variables by Shapiro-Wilk and by skewness and kurtosis to determine appropriate parametrical or non-parametrical analyses. Descriptive statistics are presented as mean and SD for parametrical data or median and IQR for non-parametrical data. To compare SLE and controls, Student's t-test was used for parametrical data and Mann-Whitney U test was used for non-parametrical data. Standardised univariate and multivariate regressions were performed and $\beta$-coefficients and $p$ values were reported. Bonferoni correction was reported for multiple comparisons.

\section{RESULTS}

SLE subjects display an atherogenic lipoprotein particle profile

Demographic and clinical characteristics of this cohort have been recently described (table 1 ). ${ }^{14}$ No patient included in the study had nephrotic range proteinuria or proteinuria $>500 \mathrm{mg} / \mathrm{dL}$. There were no differences in total cholesterol or triglyceride levels between SLE and controls. However, SLE subjects demonstrated significantly lower levels of HDL cholesterol $(54.1 \pm 16$ $\mathrm{mg} / \mathrm{dL}$ SLE vs $66.9 \pm 16 \mathrm{mg} / \mathrm{dL}$ control, $\mathrm{p}<0.001)$ and smaller HDL particle size $(9.6 \mathrm{~nm}(9.2-9.9 \mathrm{~nm})$ SLE vs $9.9 \mathrm{~nm}(9.5-10.1 \mathrm{~nm})$ control, $\mathrm{p}=0.013)$ compared with controls (table 2). HDL particle counts were significantly lower in SLE compared with controls $(30.0 \pm 5.9$ umol/L vs $35.0 \pm 7.7 \mathrm{umol} / \mathrm{L}$, respectively, $\mathrm{p}<0.001$ ), and these differences seemed to be accounted by significant decreases in medium and large HDL particle counts in SLE (table 2). LDL cholesterol levels were not significantly different between SLE and controls, but LDL particle count was elevated in SLE $(1094.0 \mathrm{nmol} / \mathrm{L}$ (871-1220 nmol/L) SLE vs $843.5 \mathrm{nmol} / \mathrm{L}$ (675-1219 $\mathrm{nmol} / \mathrm{L}$ ) control, $\mathrm{p}=0.029)$. This elevation appeared to be driven by significant increases in small LDL particle count $(419.5 \mathrm{nmol} / \mathrm{L}(211-653 \mathrm{nmol} / \mathrm{L})$ SLE vs 271.0 $\mathrm{nmol} / \mathrm{L}(0-389 \mathrm{nmol} / \mathrm{L})$ control, $\mathrm{p}=0.027)$. There were no significant differences in LDL particle size between SLE and controls. SLE subjects demonstrated elevated VLDL triglycerides $(59.9 \mathrm{mg} / \mathrm{dL}(44-87 \mathrm{mg} / \mathrm{dL}) \mathrm{SLE}$ vs $47.8 \mathrm{mg} / \mathrm{dL}(36-58 \mathrm{mg} / \mathrm{dL})$ control, $\mathrm{p}=0.031)$ and a trend towards increased VLDL particle counts (41.1 $\mathrm{nmol} / \mathrm{L}(29-61 \mathrm{nmol} / \mathrm{L})$ SLE vs $33.3 \mathrm{nmol} / \mathrm{L}$ (21-47 $\mathrm{nmol} / \mathrm{L}$ ) control, $\mathrm{p}=0.067)$. These differences seemed to be accounted by significant increases in medium and large medium VLDL particle counts (table 2). SLE subjects also demonstrated significantly decreased intermediate-density lipoprotein particle counts compared with controls $(53.5 \mathrm{nmol} / \mathrm{L}(25-124 \mathrm{nmol} / \mathrm{L})$ SLE vs $130.5 \mathrm{nmol} / \mathrm{L}(62-190 \mathrm{nmol} / \mathrm{L})$ control, $\mathrm{p}=0.001)$. There were no differences in VLDL particle size in SLE compared with controls. Overall, SLE subjects displayed a dysregulated lipoprotein particle profile.

\section{NCB significantly associates with atherogenic lipoprotein profiles in SLE}

CCTA quantified both dense calcified plaque burden and NCB, the latter being significantly elevated in SLE compared with controls $\left(86 \pm 33 \mathrm{~mm}^{2}\right.$ SLE vs $76 \pm 19 \mathrm{~mm}^{2}$ control, $\mathrm{p}<0.001)$, as recently described in this cohort. ${ }^{14}$ NCB associated with HDL subfractions whereas dense calcified plaque associated with LDL subfractions. VLDL subfractions associated with both calcified and non-calcified plaque. Specifically, NCB negatively associated with HDL cholesterol $(\beta=-0.331, \mathrm{p}=0.001)$, medium and large HDL particle count $(\beta=-0.214, p=0.029$ and $\beta=-0.292, \quad p=0.003$, respectively), and HDL particle size $(\beta=-0.226, p=0.021)$. NCB positively associated with small HDL particle count $(\beta=0.231, p=0.019)$. The 


\begin{tabular}{|c|c|c|c|}
\hline Characteristics & $\begin{array}{l}\text { Lupus } \\
\text { ( } \mathrm{N}=64)\end{array}$ & $\begin{array}{l}\text { Control } \\
(\mathrm{N}=30)\end{array}$ & $P$ values \\
\hline \multicolumn{4}{|l|}{ Demographics } \\
\hline Age (years) & $45 \pm 12$ & $37 \pm 11$ & $<0.001$ \\
\hline Female gender, $\mathrm{N}(\%)$ & $56(88 \%)$ & $29(97 \%)$ & 0.15 \\
\hline Type-2 DM & $4(6 \%)$ & $0(0 \%)$ & 0.25 \\
\hline Hyperlipidaemia & $11(17 \%)$ & $0(0 \%)$ & 0.02 \\
\hline Hypertension & 37 (58\%) & $0(0 \%)$ & $<0.001$ \\
\hline Statin use & $6(9 \%)$ & $0(0 \%)$ & 0.12 \\
\hline \multicolumn{4}{|l|}{ Race } \\
\hline Caucasian & 25 (39\%) & $16(53 \%)$ & 0.69 \\
\hline African-American & $13(20 \%)$ & $5(17 \%)$ & \\
\hline Asian & $6(9 \%)$ & $2(7 \%)$ & \\
\hline Other & $20(31 \%)$ & $7(23 \%)$ & \\
\hline \multicolumn{4}{|l|}{ Ethnicity } \\
\hline Hispanic & 27 (42\%) & $10(33 \%)$ & 0.43 \\
\hline Non-Hispanic & 35 (55\%) & 20 (67\%) & \\
\hline
\end{tabular}

History

\begin{tabular}{|c|c|c|c|}
\hline \multicolumn{4}{|l|}{ Smoking } \\
\hline $\begin{array}{l}\text { Current tobacco use, } \\
\mathrm{N}(\%)\end{array}$ & $4(7 \%)$ & $0(0 \%)$ & 0.22 \\
\hline $\begin{array}{l}\text { Previous smoker, } \mathrm{N} \\
(\%)\end{array}$ & $7(11 \%)$ & $4(14 \%)$ & 0.51 \\
\hline Physical activity, N (\%) & $19(42 \%)$ & $9(50 \%)$ & 0.90 \\
\hline \multicolumn{4}{|c|}{ Lupus history } \\
\hline Disease duration (years) & $15 \pm 12$ & - & - \\
\hline SLEDAI & $3.8 \pm 3.0$ & - & - \\
\hline SLICC & $2(0-3)$ & - & \\
\hline $\begin{array}{l}\text { History of thrombotic } \\
\text { event }\end{array}$ & $13(20 \%)$ & - & - \\
\hline \multicolumn{4}{|l|}{ Medications } \\
\hline Hydroxychloroquine & $57(89 \%)$ & - & - \\
\hline Azathioprine & $16(25 \%)$ & - & - \\
\hline Methotrexate & $10(16 \%)$ & - & - \\
\hline $\begin{array}{l}\text { Mycophenolate } \\
\text { mofetil }\end{array}$ & $19(30 \%)$ & - & - \\
\hline Prednisone & $48(75 \%)$ & - & - \\
\hline \multicolumn{4}{|l|}{ Clinical parameters } \\
\hline BMI & $28.4 \pm 6.2$ & $24.1 \pm 4.4$ & $<0.001$ \\
\hline Framingham Risk Score & $0(0-1)$ & $0(0-0)$ & 0.14 \\
\hline Glucose (mg/dL) & $90.1 \pm 13.0$ & $92.0 \pm 9.4$ & 0.23 \\
\hline Insulin (mcU/ml) & $15.0(9-20)$ & $8.0(6-11)$ & $<0.001$ \\
\hline HOMA-IR & $\begin{array}{l}3.3(1.9- \\
4.6)\end{array}$ & $1.7(1.4-2.5)$ & 0.002 \\
\hline $\begin{array}{l}\text { C reactive protein } \\
(\mathrm{mg} / \mathrm{L})\end{array}$ & $\begin{array}{l}1.6(0.8- \\
3.9)\end{array}$ & $1.1(0.7-3.2)$ & 0.18 \\
\hline Urine creatinine (mg/dl) & $\begin{array}{l}110(66- \\
181)\end{array}$ & $\begin{array}{l}133(52- \\
166)\end{array}$ & 0.90 \\
\hline
\end{tabular}

Continued

\begin{tabular}{llll} 
Table 1 Continued & & & \\
Characteristics & $\begin{array}{l}\text { Lupus } \\
(\mathbf{N}=64)\end{array}$ & $\begin{array}{l}\text { Control } \\
(\mathbf{N}=30)\end{array}$ & P values \\
\hline Urine protein $(\mathrm{mg} / \mathrm{dl})$ & $32(18-44)$ & $13(11-17)$ & $<0.001$ \\
Protein/creatinine ratio & $\begin{array}{l}0.2(0.2- \\
0.4)\end{array}$ & $0.1(0.1-0.1)$ & $<0.001$ \\
WBC count & $5.1(3.9-$ & $5.2(4.5-6.4)$ & 0.53 \\
& $6.4)$ & & \\
Neutrophil \% & $61 \pm 12$ & $56 \pm 10$ & $\mathbf{0 . 0 3}$
\end{tabular}

$\mathrm{BMI}$, body mass index; BP, blood pressure; DM, diabetes mellitus; HOMA-IR, homoeostatic model assessment insulin resistance; SLEDAI, SLE Disease Activity Index; SLICC, Systemic Lupus International Collaborating Committee.

association of HDL particle size and small and medium HDL particle counts persisted after multivariate regressions adjusting for Framingham risk (table 3). The association of NCB with HDL cholesterol and large HDL particle count also persisted after multivariate regressions adjusting for Framingham risk, HOMA-IR, past 20-day cumulative prednisone dose and SLE Disease Activity Index (SLEDAI) (table 3). NCB positively associated with large VLDL particle count $(\beta=0.277$, $\mathrm{p}=0.004)$ and VLDL particle size $(\beta=0.283, \mathrm{p}=0.004)$ that remained significant in multivariate models (table 3 ). Associations adjusted for race, ethnicity and BMI are described in table 3. Dense calcified plaque positively associated with LDL cholesterol $(\beta=0.378, p=<0.001)$ and total LDL particle count $(\beta=0.352, p=<0.001)$; the association was driven primarily by large LDL particle counts $(\beta=0.336, p=<0.001)$. The association of dense calcified plaque with LDL cholesterol, total LDL particle count and large LDL particle count persisted after multivariate regressions adjusting for Framingham risk, HOMA-IR and past 20-day cumulative prednisone dose. Dense calcified plaque positively associated with overall VLDL particle count $(\beta=0.220, p=0.025)$; this association was driven by small VLDL particle count $(\beta=0.329$, $\mathrm{p}=0.001)$, and negatively associated with VLDL particle size $(\beta=-0.238, p=0.015)$. As previously reported, the Agatston Score for coronary calcification had no associations with lipoprotein particle subfractions. ${ }^{9}$

\section{GlycA is elevated in SLE and displays significant associations with NCB}

Circulating GlycA was significantly increased in SLE (table 2) and positively associated with IR $(\beta=0.407$, $\mathrm{p}=0.001)$, Systemic Lupus International Collaborating Committee (SLICC) $(\beta=0.331, p=0.010)$, erythrocyte sedimentation rate $(\beta=0.611, p=<0.001)$ and $\mathrm{NCB}$ $(\beta=0.198, p=0.044)$ (table 4 and table 5$)$. The association between GlycA and NCB persisted after multivariate regression analysis adjusting for Framingham risk and lipoproteins, suggesting that the association of GlycA and NCB is independent of the association that lipoproteins showed with plaque (table 3 ). As previously 
Table 2 Lipoprotein particle subfractions and GlycA in SLE and healthy controls as assessed by NMR

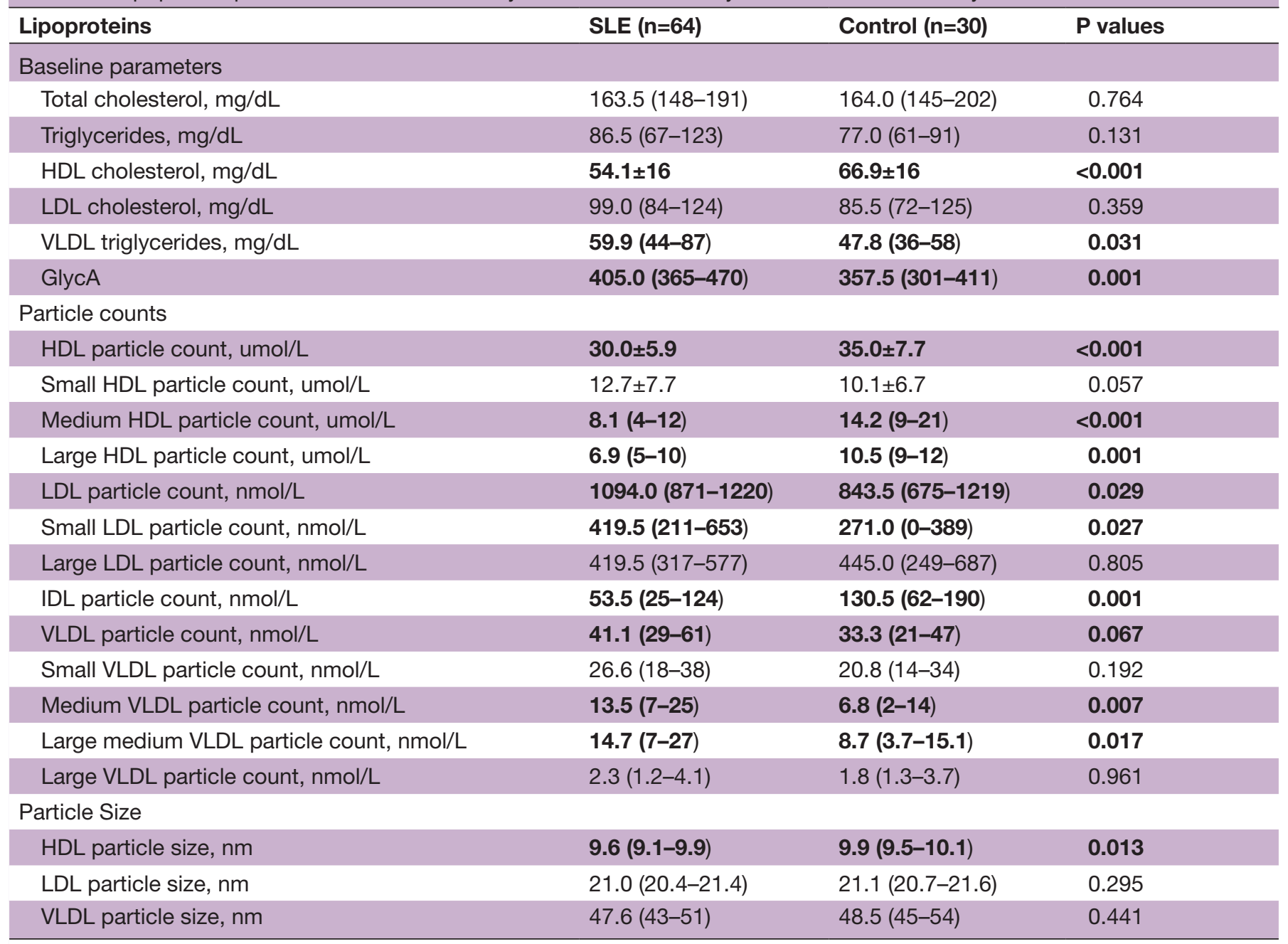

Student's t-test used unless otherwise noted (mean \pm SD).

Parameters reaching significance $(p \leq 0.05)$ highlighted in bold; after controlling for multiple comparisons only those with $p<0.00009$ remain significant.

*Mann-Whitney test used (median (IQR)).

GlycA, glycoprotein acetylation; HDL, high-density lipoprotein; IDL, intermediate-density lipoprotein; LDL, low-density lipoprotein; NMR, nuclear MR; VLDL, very low-density lipoprotein.

reported, GlycA associated with a cumulative prednisone dose in a univariate regression and no longer associated with NCB when prednisone was added to the multivariate model (tables 2 and 3). ${ }^{15}$ To determine if GlycA could provide value beyond current markers of inflammation, it was compared with high-sensitivity $\mathrm{C}$ reactive protein (hsCRP) in univariate regressions associating these markers with disease parameters. While hsCRP associated better with disease duration and traditional risk factors of atherosclerosis (Framingham Risk), GlycA was a better predictor than hsCRP of IR, SLICC, total plaque burden and NCB (table 4).

Associations of SLE disease markers with lipoprotein profiles SLEDAI, a measure of recent or current disease activity, was negatively associated with HDL particle count $(\beta=-0.354, p=0.006)$ and, in particular, with small HDL particle counts $(\beta=-0.281, p=0.026)$ (table 5 and table 6$)$.
The SLICC Disease Index, a measure of accrued damage over time, did not associate with the lipoprotein profile. Protein:creatinine ratio in SLE was positively associated with VLDL triglycerides $(\beta=0.280, p=0.038)$ and VLDL particle counts $(\beta=0.436, p=0.001)$, particularly small VLDL particle counts $(\beta=0.498, \mathrm{p}=<0.001)$ (table 5 and table 6). C3 complement levels positively associated with small HDL particle counts $(\beta=0.377, \mathrm{p}=0.002)$, total LDL particle counts $(\beta=0.332, p=0.008)$ and small LDL particle counts $(\beta=0.320, p=0.010)$, and negatively associated with large HDL particle counts $(\beta=-0.333$, $\mathrm{p}=0.008)$ and HDL particle size $(\beta=-0.456, p=<0.001)$ (table 6). C4 complement levels were positively associated with small HDL particle count $(\beta=0.259, p=0.040)$. Anti-double stranded (ds) DNA and anti-extractable nuclear antigen (ENA) antibody levels negatively associated with overall HDL particle counts $(\beta=-0.264$, 


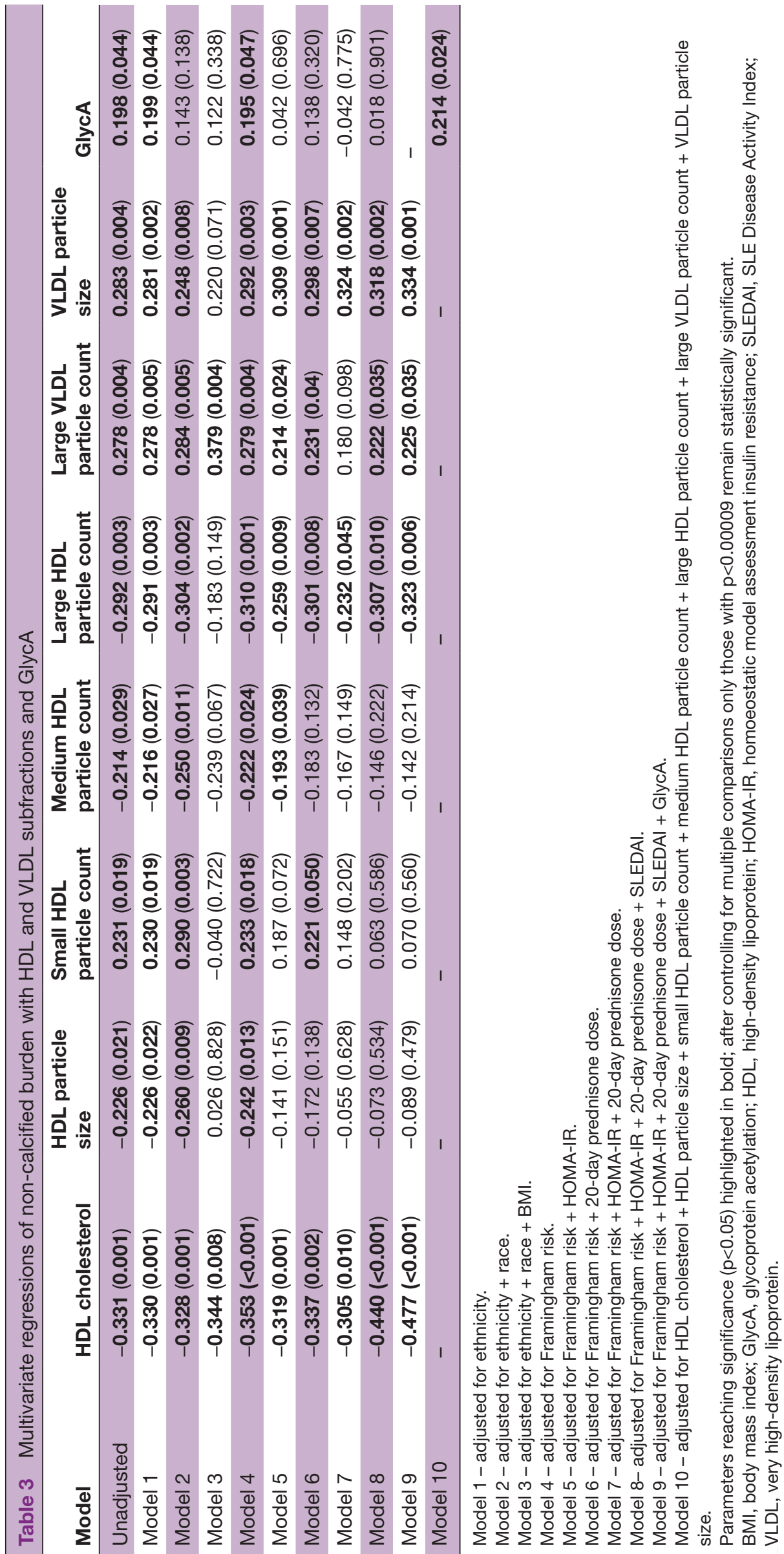




\begin{tabular}{|c|c|c|}
\hline Parameter & GlycA ( $n=64)$ & hsCRP ( $n=63)$ \\
\hline \multicolumn{3}{|l|}{$\begin{array}{l}\text { Demographic and clinical } \\
\text { characteristics }\end{array}$} \\
\hline Age & $0.013(0.901)$ & $0.199(0.118)$ \\
\hline Gender & $0.011(0.934)$ & $0.090(0.485)$ \\
\hline Smoking, N (\%) & $0.240(0.060)$ & $0.534(<0.001)$ \\
\hline \multicolumn{3}{|l|}{$\begin{array}{l}\text { Clinical and laboratory } \\
\text { values }\end{array}$} \\
\hline BMI, $\mathrm{kg} / \mathrm{m}^{2}$ & $0.328(0.008)$ & $0.221(0.081)$ \\
\hline Systolic BP, mm Hg & $0.033(0.794)$ & $0.052(0.687)$ \\
\hline Diastolic BP, mm Hg & $0.023(0.854)$ & $-0.155(0.225)$ \\
\hline Total cholesterol, mg/dL & $-0.008(0.950)$ & $-0.127(0.322)$ \\
\hline LDL, mg/dL & $0.008(0.952)$ & $-0.051(0.695)$ \\
\hline $\mathrm{HDL}, \mathrm{mg} / \mathrm{dL}$ & $-0.139(0.272)$ & $-0.226(0.075)$ \\
\hline Triglycerides, mg/dL & $0.187(0.143)$ & $0.043(0.739)$ \\
\hline Framingham Risk Score & $0.040(0.752)$ & $0.310(0.013)$ \\
\hline HOMA-IR & $0.407(0.001)$ & $0.234(0.065)$ \\
\hline Glucose, mg/dL & $0.270(0.031)$ & $0.093(0.470)$ \\
\hline Insulin, mcU/ml & $0.433(<0.001)$ & $0.247(0.051)$ \\
\hline $\begin{array}{l}\text { Erythrocyte sedimentation } \\
\text { rate, } \mathrm{mm} / \text { hour }\end{array}$ & $0.611(<0.001)$ & $0.284(0.025)$ \\
\hline
\end{tabular}

High-sensitivity $\mathrm{C}$ reactive $0.578(<0.001)$ protein, $\mathrm{mg} / \mathrm{L}$

\begin{tabular}{|c|c|c|}
\hline Protein/creatinine ratio & $0.226(0.097)$ & $-0.071(0.611)$ \\
\hline WBC count & $0.441(<0.001)$ & $0.233(0.068)$ \\
\hline \multicolumn{3}{|l|}{ SLE characteristics } \\
\hline SLEDAI & $0.011(0.934)$ & $0.043(0.740)$ \\
\hline SLICC & $0.331(0.010)$ & $0.293(0.024)$ \\
\hline Disease duration, years & $0.226(0.073)$ & $0.456(<0.001)$ \\
\hline $\begin{array}{l}\text { Hydroxychloroquine, N } \\
(\%)\end{array}$ & $0.079(0.533)$ & $0.026(0.841)$ \\
\hline Azathioprine, N (\%) & $0.205(0.104)$ & $-0.092(0.471)$ \\
\hline Methotrexate, N (\%) & $-0.187(0.140)$ & $-0.014(0.911)$ \\
\hline $\begin{array}{l}\text { Mycophenolate mofetil, } \\
\mathrm{N}(\%)\end{array}$ & $-0.043(0.735)$ & $-0.114(0.372)$ \\
\hline $\begin{array}{l}\text { Cumulative } 20 \text {-day } \\
\text { steroid dose, mg }\end{array}$ & $0.410(0.016)$ & $-0.178(0.314)$ \\
\hline \multicolumn{3}{|l|}{ Coronary plaque burden } \\
\hline Total burden, $(\times 100) \mathrm{mm} 2$ & $0.217(0.027)$ & $-0.131(0.184)$ \\
\hline $\begin{array}{l}\text { Calcified burden, }(\times 100) \\
\mathrm{mm}^{2}\end{array}$ & $0.092(0.355)$ & $-0.077(0.438)$ \\
\hline $\begin{array}{l}\text { Non-calcified burden, } \\
(\times 100) \mathrm{mm}^{2}\end{array}$ & $0.198(0.044)$ & $-0.117(0.238)$ \\
\hline
\end{tabular}

Univariate regressions reported as $\beta$-coefficient ( $p$ value). Parameters reaching significance $(p \leq 0.05)$ highlighted in bold; after controlling for multiple comparisons only those with $\mathrm{p} \leq 0.00009$ remain statistically significant.

$\mathrm{BMI}$, body mass index; GlycA, glycoprotein acetylation; HDL, high-density lipoprotein; HOMA-IR, homoeostatic model assessment insulin resistance; LDL, low-density lipoprotein; SLEDAI, SLE Disease Activity Index; SLICC, Systemic Lupus International Collaborating Committee; WBC, white blood cell count; hsCRP, high-sensitivity $C$ reactive protein. $p=0.037$ and $\beta=-0.273, p=0.032$, respectively). Anti-La autoantibodies negatively associated with large HDL particle counts $(\beta=-0.262, p=0.043)$, while anti-ribonuclear protein (RNP) autoantibodies negatively associated with both total HDL particle count $(\beta=-0.295$, $\mathrm{p}=0.022)$, and LDL particle size $(\beta=-0.273, \mathrm{p}=0.035)$ (table 6). Anti-Smith antibodies and SLE disease duration demonstrated no associations with lipoprotein particle subfractions. A past 20-day prednisone dose did associate with NMR parameters, however other medications did not show associations (table 7). Overall, dysregulation in lipoprotein fractions by NMR was significantly associated with lupus activity and specific autoantibody profiles. Of note, only $\mathrm{p}$ values less than 0.00009 in this study are statistically significant after controlling for multiple comparisons.

\section{DISCUSSION}

We report that SLE subjects with overall mild to moderate disease activity display a proatherogenic lipoprotein profile characterised by lower levels and smaller size of HDL particles and increases in VLDL and LDL particle number. Our findings are in agreement with previous studies that have shown decreases in HDL and increases in VLDL particle counts and triglycerides in SLE. ${ }^{16}$ As previously suggested, our findings indicate that lupus disease activity is associated with a more proatherogenic lipid profile. $^{16}$

To our knowledge, this is the first study to associate the aberrant lipoprotein profile in SLE with burden of non-calcified coronary plaque. The shift towards smaller HDL particle size in SLE could support a proatherogenic environment that contributes to the enhanced NCB in SLE. This is significant because NCB can predict CV events in other patient populations and is considered higher risk for unstable plaque development. ${ }^{10-12}$ Although we observed a significant association between LDL particles and dense calcified plaque in fully adjusted models, we did not see an association between LDL and NCB. This is in contrast to previous studies that have identified LDL as a predictor of NCB in other patient populations. ${ }^{17} 18$ Those studies were not focused on patients with autoimmune conditions, suggesting that the pathways underlying atherogenesis in SLE could be distinct. Our group has previously reported that modifications to HDL in SLE cause it to become proinflammatory and atherogenic due to its impaired cholesterol efflux capacity (CEC).$^{19}$ Indeed, small HDL particle numbers associate with impaired CEC and we recently found that impaired CEC associates with NCB in SLE. ${ }^{14} 20$

An association between VLDL and plaque burden, measured by carotid intima-media thickness, was previously reported in SLE. ${ }^{21}$ Here, we found that large VLDL particle counts and VLDL particle size positively associated with both NCB and calcified plaque, providing additional evidence that VLDL may act as an independent predictor of subclinical coronary artery disease in SLE. It has been 


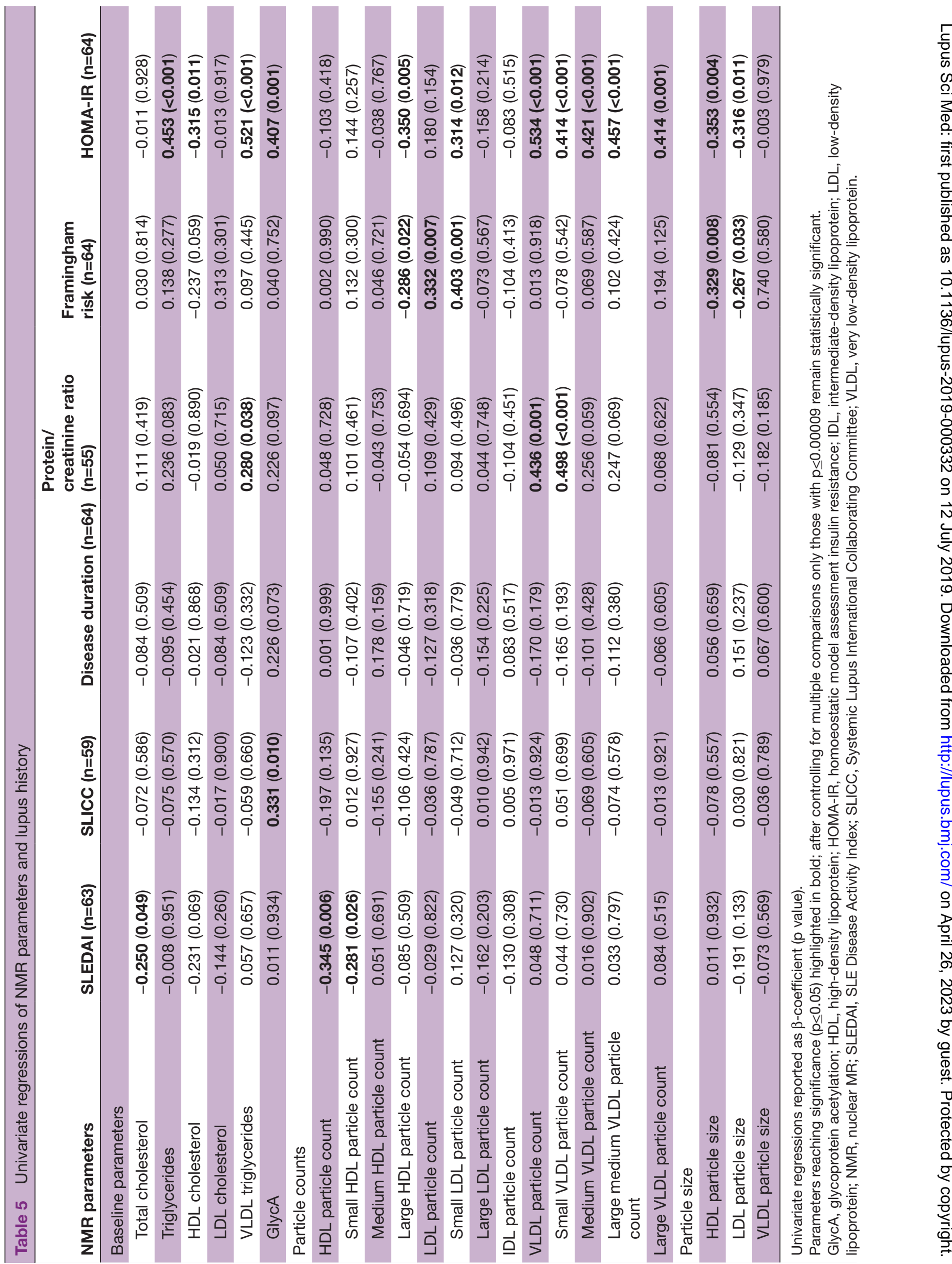




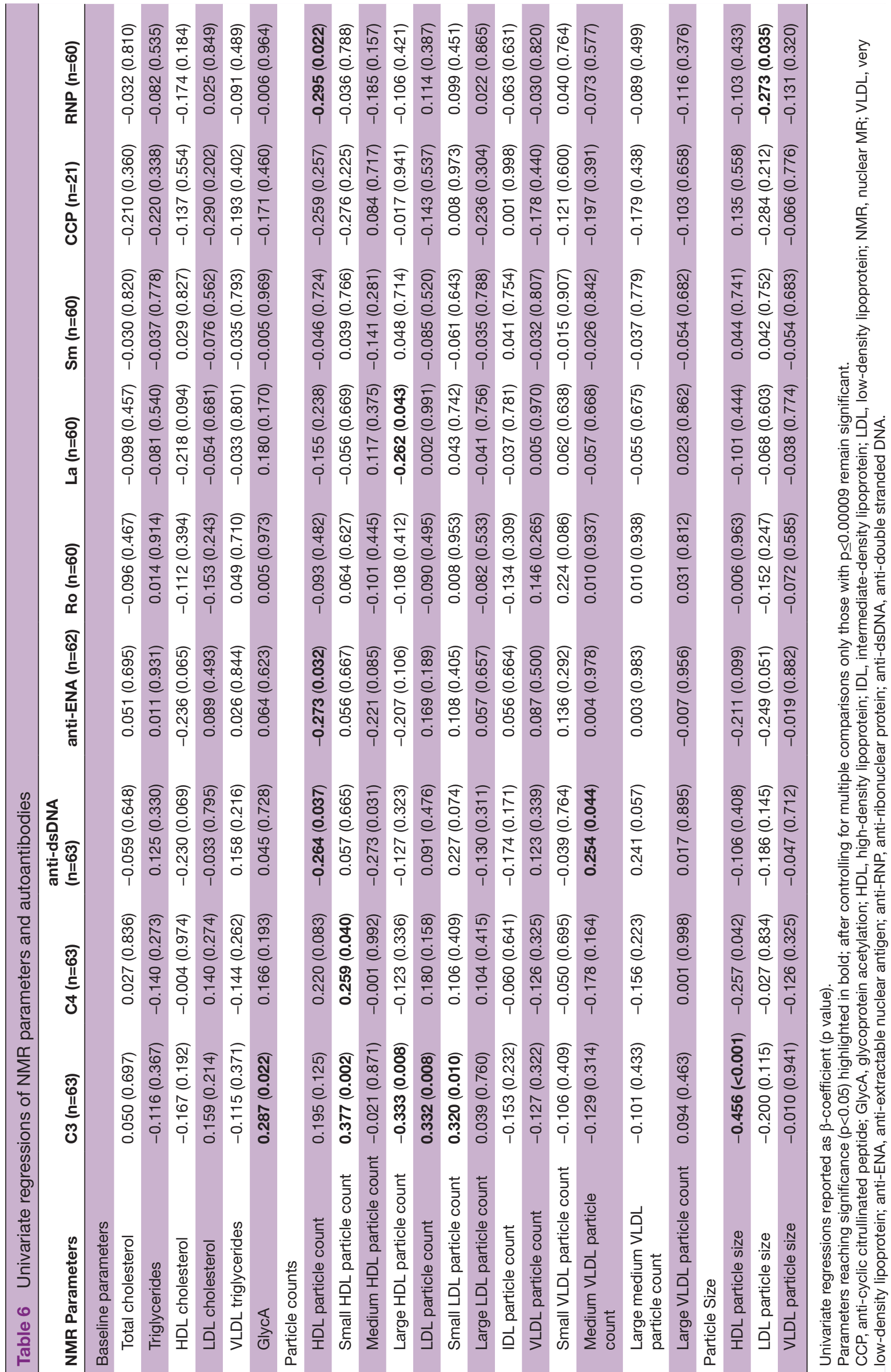


Table 7 Univariate regressions of NMR parameters and medications

\begin{tabular}{|c|c|c|c|c|}
\hline NMR parameters & Prednisone $(n=64)$ & $\begin{array}{l}\text { 20-day prednisone } \\
\text { dose }(n=34)\end{array}$ & $\begin{array}{l}\text { Hydroxychloroquine } \\
(\mathrm{n}=64)\end{array}$ & Statins $(n=64)$ \\
\hline \multicolumn{5}{|l|}{ Baseline parameters } \\
\hline Total cholesterol & $0.045(0.724)$ & $0.243(0.166)$ & $0.082(0.519)$ & $-0.042(0.740)$ \\
\hline Triglycerides & $0.143(0.261)$ & $0.405(0.018)$ & $0.178(0.160)$ & $-0.133(0.294)$ \\
\hline HDL cholesterol & $0.048(0.707)$ & $-0.021(0.904)$ & $0.009(0.946)$ & $0.123(0.333)$ \\
\hline LDL cholesterol & $0.013(0.918)$ & $0.333(0.054)$ & $0.078(0.542)$ & $-0.107(0.398)$ \\
\hline VLDL triglycerides & $0.162(0.200)$ & $0.430(0.011)$ & $0.172(0.174)$ & $-0.150(0.236)$ \\
\hline GlycA & $0.214(0.089)$ & $0.410(0.016)$ & $0.079(0.553)$ & $0.133(0.294)$ \\
\hline \multicolumn{5}{|l|}{ Particle counts } \\
\hline HDL particle count & $-0.217(0.086)$ & $-0.206(0.243)$ & $-0.067(0.596)$ & $0.136(0.285)$ \\
\hline Small HDL particle count & $-0.236(0.060)$ & $0.049(0.784)$ & $-0.111(0.384)$ & $-0.034(0.790)$ \\
\hline Medium HDL particle count & $-0.079(0.536)$ & $-0.374(0.029)$ & $0.033(0.795)$ & $0.104(0.415)$ \\
\hline Large HDL particle count & $0.200(0.112)$ & $0.048(0.787)$ & $0.050(0.694)$ & $0.121(0.341)$ \\
\hline LDL particle count & $-0.050(0.694)$ & $0.321(0.065)$ & $0.046(0.717)$ & $-0.135(0.289)$ \\
\hline Small LDL particle count & $-0.114(0.370)$ & $0.025(0.889)$ & $-0.027(0.834)$ & $-0.088(0.488)$ \\
\hline Large LDL particle count & $0.046(0.716)$ & $0.338(0.050)$ & $0.112(0.380)$ & $-0.038(0.764)$ \\
\hline IDL particle count & $0.124(0.327)$ & $0.011(0.951)$ & $-0.028(0.826)$ & $-0.083(0.516)$ \\
\hline VLDL particle count & $0.179(0.157)$ & $0.468(0.005)$ & $0.115(0.365)$ & $-0.114(0.368)$ \\
\hline Small VLDL particle count & $0.136(0.283)$ & $0.340(0.049)$ & $0.093(0.467)$ & $-0.027(0.831)$ \\
\hline Medium VLDL particle count & $0.172(0.173)$ & $0.419(0.014)$ & $0.074(0.561)$ & $-0.162(0.202)$ \\
\hline Large medium VLDL particle count & $0.156(0.219)$ & $0.424(0.013)$ & $0.095(0.453)$ & $-0.161(0.203)$ \\
\hline Large VLDL particle count & $0.033(0.793)$ & $0.194(0.271)$ & $0.183(0.149)$ & $-0.048(0.706)$ \\
\hline \multicolumn{5}{|l|}{ Particle size } \\
\hline HDL particle size & $0.184(0.147)$ & $-0.063(0.725)$ & $0.036(0.778)$ & $0.069(0.588)$ \\
\hline LDL particle size & $0.063(0.619)$ & $0.003(0.985)$ & $0.121(0.342)$ & $0.086(0.498)$ \\
\hline VLDL particle size & $0.094(0.459)$ & $0.112(0.529)$ & $0.081(0.523)$ & $0.022(0.863)$ \\
\hline
\end{tabular}

Univariate regressions reported as $\beta$-coefficient ( $p$ value).

Parameters reaching significance $(p \leq 0.05)$ highlighted in bold; after controlling for multiple comparisons only those with $p \leq 0.00009$ remain statistically significant.

GlycA, glycoprotein acetylation; HDL, high-density lipoprotein; IDL, intermediate-density lipoprotein; LDL, low-density lipoprotein; NMR, nuclear MR; VLDL, very low-density lipoprotein.

suggested that decreased lipoprotein lipase activity, potentially due to autoantibodies targeting this enzyme in SLE, promotes lipoprotein dysregulation and increased VLDL and LDL levels. ${ }^{22}$ Given the associations identified here between VLDL and NCB, future studies should investigate the role that lipoprotein lipase has in VLDL deposition and atherosclerotic progression. In addition, traditional clinical laboratory tests do not capture the detailed assessments of lipoprotein subfraction counts and sizes. Our data suggest that, in SLE, small HDL particles have a positive association with proatherogenic pathways while the opposite is observed with larger HDL particles. It may therefore be beneficial for clinicians to determine the relative abundance of small HDL particles when determining potential CV risk in patients with SLE. This reinforces the notion that using NMR to obtain a detailed assessment of lipoprotein parameters may help better characterise CVD risk in lupus.
In other patient populations, GlycA confers additional value beyond traditional biomarkers of inflammation, like hsCRP, in predicting long-term CV and all-cause mortality. ${ }^{8}$ In support of this, GlycA but not hsCRP was significantly elevated in SLE. Although this increase in GlycA has been shown to predict systemic inflammation in SLE, its association to subclinical coronary artery disease had not been identified because of the reliance on coronary calcification scores. ${ }^{23}$ We now found that GlycA associates with NCB but not with calcified plaque. Moreover, a comparison between GlycA and hsCRP revealed that GlycA significantly associated with IR and plaque burden in SLE, while hsCRP did not. Taken together, our findings suggest that GlycA may be a better tool to assess CV risk in SLE than hsCRP. Indeed, previous studies have shown that CRP is suppressed by type I interferons and this may explain the poor association of this acute 
phase reactant to lupus disease activity and organ-specific complications. ${ }^{24}$

Although GlycA represents a composite NMR signal of multiple acetylated glycoproteins, it has been suggested that neutrophils are an important source of two major protein contributors to the GlycA signal, $\alpha 1$-acid glycoprotein and haptoglobin. ${ }^{8}$ It has also been shown that elevated GlycA is associated with neutrophil activity. ${ }^{8}$ In this cohort, we have previously identified elevated levels of a pathogenic neutrophil subset, known as low density granulocytes, which display an activated phenotype and associate with NCB in SLE. ${ }^{14}$ These neutrophils could potentially serve as a significant source for the elevated GlycA in SLE and the extent to which they contribute to the GlycA signal should be investigated. The association of GlycA with NCB did not persist after controlling for prednisone dose. These findings suggest that there is a possible link between GlycA and corticosteroid use and that GlycA may be useful in tracking vascular damage caused by steroids. In addition, this could be a confounding effect as patients with more severe disease tend to take higher doses of steroids. The associations of GlycA with inflammatory markers and plaque imply that the inflammatory pathways producing acetylated glycoproteins may play a role in driving atherogenesis. Future studies should seek to determine the putative role that the pathways associated with this marker have in other aspects of lupus pathogenesis.

Although our study was limited by a relatively small sample size and included patients with mild to moderate disease activity, GlycA and lipoprotein profiles still independently predicted NCB in SLE. However, due to the exploratory nature of this cross-sectional analysis, after controlling for multiple comparisons, only $\mathrm{p}$ values less than 0.0009 remain statistically significant. Our study was not appropriately powered to meet this requirement and is therefore subject to type 1 error. Future studies should confirm these findings in larger cohorts and study the predictive value of GlycA and lipoprotein profiles in subjects with more severe disease. Longitudinal assessments of these parameters are also required to further understand the pathogenicity of lipoprotein subsets in SLE and the extent to which GlycA fluctuates with changes in vascular damage and predicts progression to coronary events.

Contributors MMP, PMC, SS, MS performed experiments; AKD, JBB, JHC, TS, MYC and NNM were involved in statistical analysis and/or coronary CT quantification; YT-0, SS, AF, MC, SG, SH,were involved in patient assessment and recruitment and clinical phenotyping; ZM managed the database; AR and MS were involved in interpretation of lipoprotein data; MMP, PMC and MJK designed the study and wrote the manuscript.

Funding This study was supported by the Intramural Research program at NIAMS/ NIH (ZIAAR041199) and by the Lupus Research Institute.

Competing interests None declared.

Patient consent for publication Not required.

Ethics approval The study was approved by the Institutional Review Board of National Institute of Arthritis and Muskuloskeletal and Skin Diseases/National Institute of Diabetes and Digestive and Kidney Diseases (NIAMS/NIDDK), Bethesda, Maryland, under protocol 94-AR-0066.

Provenance and peer review Not commissioned; externally peer reviewed.
Data availability statement All data relevant to the study are included in the article or uploaded as supplementary information.

Open access This is an open access article distributed in accordance with the Creative Commons Attribution Non Commercial (CC BY-NC 4.0) license, which permits others to distribute, remix, adapt, build upon this work non-commercially, and license their derivative works on different terms, provided the original work is properly cited, appropriate credit is given, any changes made indicated, and the use is non-commercial. See: http://creativecommons.org/licenses/by-nc/4.0/.

\section{REFERENCES}

1. Manzi S, Selzer F, Sutton-Tyrrell K, et al. Prevalence and risk factors of carotid plaque in women with systemic lupus erythematosus. Arthritis Rheum 1999;42:51-60.

2. Esdaile JM, Abrahamowicz M, Grodzicky T, et al. Traditional Framingham risk factors fail to fully account for accelerated atherosclerosis in systemic lupus erythematosus. Arthritis Rheum 2001;44:2331-7.

3. Remaley AT. HDL cholesterol/HDL particle ratio: a new measure of HDL function? J Am Coll Cardiol 2015;65:364-6.

4. Rosenson RS, Brewer HB, Chapman MJ, et al. HDL measures, particle heterogeneity, proposed nomenclature, and relation to atherosclerotic cardiovascular events. Clin Chem 2011;57:392-410.

5. Asanuma Y, Oeser A, Shintani AK, et al. Premature coronary-artery atherosclerosis in systemic lupus erythematosus. $N$ Engl $J$ Med 2003;349:2407-15.

6. Kuller L, Arnold A, Tracy R, et al. Nuclear magnetic resonance spectroscopy of lipoproteins and risk of coronary heart disease in the cardiovascular health study. Arterioscler Thromb Vasc Biol 2002;22:1175-80.

7. Otvos JD, Jeyarajah EJ, Bennett DW. Quantification of plasma lipoproteins by proton nuclear magnetic resonance spectroscopy. Clin Chem 1991;37:377-86.

8. Connelly MA, Otvos JD, Shalaurova I, et al. GlycA, a novel biomarker of systemic inflammation and cardiovascular disease risk. J Trans/ Med 2017;15.

9. Chung CP, Oeser A, Raggi P, et al. Lipoprotein subclasses and particle size determined by nuclear magnetic resonance spectroscopy in systemic lupus erythematosus. Clin Rheumatol 2008;27:1227-33.

10. Hoffmann U, Moselewski F, Nieman K, et al. Noninvasive assessment of plaque morphology and composition in culprit and stable lesions in acute coronary syndrome and stable lesions in stable angina by multidetector computed tomography. J Am Coll Cardiol 2006;47:1655-62.

11. Salahuddin T, Natarajan B, Playford MP, et al. Cholesterol efflux capacity in humans with psoriasis is inversely related to non-calcified burden of coronary atherosclerosis. Eur Heart J 2015;36:2662-5.

12. Nance JW, Bamberg F, Schoepf UJ, et al. Coronary atherosclerosis in African American and white patients with acute chest pain: characterization with coronary CT angiography. Radiology 2011;260:373-80.

13. Hochberg MC. Updating the American College of Rheumatology revised criteria for the classification of systemic lupus erythematosus. Arthritis Rheum 1997;40.

14. Carlucci PM, Purmalek MM, Dey AK, et al. Neutrophil subsets and their gene signature associate with vascular inflammation and coronary atherosclerosis in lupus. JCl Insight 2018;3. doi:10.1172/jci. insight.99276

15. Durcan L, Winegar DA, Connelly MA, et al. Longitudinal Evaluation of Lipoprotein Variables in Systemic Lupus Erythematosus Reveals Adverse Changes with Disease Activity and Prednisone and More Favorable Profiles with Hydroxychloroquine Therapy. J Rheumatol 2016;43:745-50.

16. Borba EF, Bonfá E. Dyslipoproteinemias in systemic lupus erythematosus: influence of disease, activity, and anticardiolipin antibodies. Lupus 1997;6:533-9.

17. Cheng VY, Wolak A, Gutstein A, et al. Low-density lipoprotein and noncalcified coronary plaque composition in patients with newly diagnosed coronary artery disease on computed tomographic angiography. Am J Cardiol 2010;105:761-6.

18. Rodriguez K, Kwan AC, Lai S, et al. Coronary Plaque Burden at Coronary CT Angiography in Asymptomatic Men and Women. Radiology 2015;277:73-80.

19. Smith CK, Vivekanandan-Giri A, Tang C, et al. Neutrophil extracellular trap-derived enzymes oxidize high-density lipoprotein: an additional 
proatherogenic mechanism in systemic lupus erythematosus. Arthritis Rheumatol 2014;66:2532-44.

20. Mutharasan RK, Thaxton CS, Berry J, et al. HDL efflux capacity, HDL particle size, and high-risk carotid atherosclerosis in a cohort of asymptomatic older adults: the Chicago Healthy Aging Study. J Lipid Res 2017;58:600-6.

21. Belibou C, Ancuța C, Ancuța E, et al. Carotid intima-media thickness and plaque as surrogate biomarkers of atherosclerosis among consecutive women with systemic lupus erythematosus. Rom J Morphol Embryol 2012;53.
22. Reiss AB. Effects of inflammation on cholesterol metabolism: impact on systemic lupus erythematosus. Curr Rheumatol Rep 2009;11:255-60.

23. Chung CP, Ormseth MJ, Connelly MA, et al. GlycA, a novel marker of inflammation, is elevated in systemic lupus erythematosus. Lupus 2016;25:296-300.

24. Enocsson H, Sjöwall C, Skogh T, et al. Interferon-alpha mediates suppression of C-reactive protein: explanation for muted C-reactive protein response in lupus flares? Arthritis Rheum 2009;60:3755-60. 